\title{
Secondary User Access in LTE Architecture Based on a Base-Station-Centric Framework With Dynamic Pricing
}

\author{
Soumitra Dixit, Member, IEEE, Shalini Periyalwar, Senior Member, IEEE, and \\ Halim Yanikomeroglu, Member, IEEE
}

\begin{abstract}
Dynamic spectrum access (DSA) techniques based on the exclusive-use model provide a huge opportunity for wireless service providers (WSPs) to improve the spectrum utilization in their licensed bands and generate additional profits by allowing temporary wireless access to unsubscribed secondary users (SUs). This paper presents a techno-economic analysis for regulated SU access based on a novel base station (BS)-centric framework, where SUs coexist with the subscribers, i.e., primary users (PUs), on a mutually exclusive basis. Considering the highly competitive WSP environment, the proposed framework is aimed at maximizing the localized spectrum utilization within the static spectrum licensed to the WSP and assumes no cooperation and no spectrum sharing among WSPs, thus making this a business case for implementation. The dynamic incentive-based SU pricing model proposed in this paper has the inherent capability of call admission control and, hence, is useful in attracting SUs to obtain temporary wireless access during periods of low PU demand, thus improving spectrum usage in the temporal domain. Although SU access is regulated by the WSP at their BS, the SUs have the freedom to connect or handoff to their preferred BS in the area based on the SU price quoted by the WSP. The implementation of the proposed framework to Long Term Evolution (LTE) infrastructure requires minimal enhancements and can be potentially attractive to WSPs, since the SU devices in this framework do not require spectrum sensing cognitive capabilities. Considering all the aforementioned aspects, this paper can be considered an intermediate step in the evolution toward complete DSA.
\end{abstract}

Index Terms-Dynamic spectrum access (DSA), Long-Term Evolution (LTE) architecture, secondary user (SU) access, SU pricing.

\section{INTRODUCTION}

$\mathbf{P}$ HENOMENAL growth in wireless technology parallel to the Internet has led to an ever-increasing demand for wireless services in certain licensed and unlicensed spectrum bands. The traditional spectrum management approach with static spectrum allocations in combination with unbalanced

Manuscript received February 9, 2011; revised April 13, 2012; accepted August 28, 2012. Date of publication October 2, 2012; date of current version January 14, 2013. This paper was presented in part at the IEEE Vehicular Technology Conference (VTC), Anchorage, AK, September 20-23, 2009 and at the IEEE VTC, Ottawa, ON, Canada, September 6-9, 2010. This work was supported by the Natural Science and Engineering Research Council of Canada Discovery Grant. The review of this paper was coordinated by Prof. R. Jäntti.

The authors are with the Department of Systems and Computer Engineering, Carleton University, Ottawa, ON K1S 5B6, Canada (e-mail: sdixit@sce.carleton.ca; shalinip@ sce.carleton.ca; halim@ @sce.carleton.ca).

Color versions of one or more of the figures in this paper are available online at http://ieeexplore.iee.org.

Digital Object Identifier 10.1109/TVT.2012.2221753 utilization in the different spectrum bands has created a "pseudo" scarcity of the available radio spectrum. With the goal of improving the efficiency of spectrum utilization, the Spectrum Policy Task Force released a report [1] promoting the design and development of flexible spectrum sharing techniques under the dynamic spectrum access (DSA) paradigm.

This new DSA paradigm gave rise to secondary markets, where secondary users (SUs) can gain opportunistic access to the white spaces in the spectrum available instantaneously at that time and location. To allow such opportunistic SU access, several techniques have been proposed in the literature based on two primary models: 1) the "exclusive-use" model, where SU access needs to be regulated for operation in licensed bands, and 2) the "commons" model, where a general "etiquette" needs to be maintained by all the SUs operating in the unlicensed bands. Focusing on the "exclusive-use" model, efficient utilization of the licensed spectrum is of critical importance to wireless service providers (WSPs), considering the huge costs involved in CAPital EXpenditure (CAPEX) and OPerational EXpenditure (OPEX) for spectrum licensing and infrastructure [2], [3].

\section{A. Proposed Framework: Rationale}

WSPs, or licensed band owners, have been apprehensive about the implementation of DSA in their networks, considering the possible impact of interference to their subscribers due to such opportunistic SU access. Additionally, mechanisms for spectrum sharing in the highly competitive WSP space can also cause legal concerns, due to the possible infringements in shortand long-term spectrum license transfers [2], [3].

Our approach toward DSA in this paper is fairly conservative and is primarily in line with the current status of the available wireless technology. Since this framework is not based on opportunistic SU access, the SU devices are not required to have cognitive capabilities for spectrum sensing or interference management. The SU devices are assumed to be at par with current cellphone technology with the capability to support multiple radio access technologies (RATs) in the licensed bands. From the WSP perspective, considering the high amount of competition observed in the WSP space, the proposed framework assumes no spectrum sharing and no inter-WSP cooperation. Therefore, the proposed framework can be considered an intermediate step toward transformation to a more complete DSA architecture. 


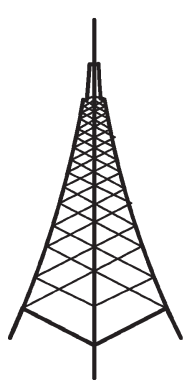

$\mathrm{BS}_{i}$ of $\mathrm{WSP}_{w}$
Single Frequency Network: Frequency band licensed by $\mathrm{WSP}_{\mathrm{w}}$

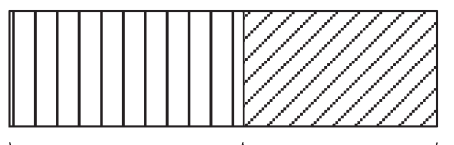

\section{Spectrum} utilized by the PUs

$\left(\alpha_{i, w}^{p u}\right)$
Unutilized spectrum available for SU access $\left(1-\alpha_{i, w}^{p u}\right)$
Fig. 1. BS-centric framework and spectrum utilization.

\section{B. Proposed Framework: System Concept and Key Features}

The proposed framework is base station (BS) centric, such that the BS regulates temporary wireless access for SUs to the instantaneously unutilized spectrum in the WSP licensed band without affecting the current subscriber, i.e., primary user (PU), service. The process is initiated with the BS advertising the currently unutilized spectrum to an SU in the area for a fixed fee per service type, as shown in Fig. 1. The SU then sends a request to the $\mathrm{BS}$ indicating the service type (application class) it wishes to access. Finally, the BS authenticates the SU and provides the SU with temporary wireless access on a mutually exclusive resource, thus avoiding interference to PU service. The key idea behind the proposed framework is to achieve a strategic change in SU access behavior through price-based incentives, such that SUs will prefer to obtain temporary wireless access primarily during off-peak hours. This is achievable through the dynamic incentive-based SU pricing model, which is based on the PU demand. Thus, during periods of high PU demand, the SU price is significantly high, which helps deter the SUs from wanting to gain temporary access during peak hours. During the periods of lower PU demand, the dynamic pricing model increments the price for every subsequent $\mathrm{SU}$ joining the BS for temporary access, thus providing inherent SU admission control. Additionally, the proposed framework will be shown to require only minimal modifications to existing wireless infrastructures, e.g., Long-Term Evolution (LTE), and, therefore, can be a viable option for implementation by the WSPs.

From the SU perspective, this framework not only provides the SUs with the freedom to select the preferred BS in the area based on price but also gives them monetary incentives to gain temporary wireless access during periods of low PU demand. With minimal enhancements required for the implementation of the proposed framework in existing wireless infrastructures (e.g., LTE), the WSPs can thus benefit by keeping their investments low and gaining additional profits from such SU access.

The four key contributions in this paper are as follows:

- proposed BS-centric distributed framework for SU access, such that PUs and SUs coexist and PU service is not affected by SU access;

- implementation of the proposed framework to $4 \mathrm{G}$ LTE architecture for such SU access;
- SU-centric BS selection and price-based handoff scheme;

- dynamic incentive-based pricing model for charging SUs, with extension to multiple WSPs competitive environment.

The various contributions of this paper together constitute a joint techno-economic analysis for SU access in the proposed distributed framework.

This paper is organized as follows. A brief review of the literature is presented in Section II, followed by the system model and an SU-initiated network (BS) selection along with a price-based handoff scheme in Section III. Section IV then introduces the novel dynamic incentive-based SU pricing model for additional revenue generation from $\mathrm{SU}$ access considering a single BS of a single WSP. Section V enhances the dynamic SU incentive-based pricing model for single WSP to a competitive SU pricing structure in multiple WSP environment. Section VI describes an overview on the implementation of the proposed framework to 4G LTE architecture, followed by the conclusion in Section VII.

\section{Literature REVIEW}

The exclusive-use model, as defined in [1], led the way for the research and development of various spectrum sharing techniques in the licensed bands [2], [3]. Here, we present a comparative study of the proposed work with respect to other works in the literature.

With respect to SU devices, the proposed framework does not require the SU devices to possess cognitive capabilities, as opposed to the majority of the works in the literature that rely on the cognitive abilities of SU devices for sensing white spaces in the spectrum, or detecting and vacating the spectrum band in the presence of PUs, or avoiding and minimizing interference to PUs [2], [3]. Considering the spectrum sharing aspect, plenty of works in the literature consider cooperation among WSPs for spectrum sharing [4]-[6] and/or spectrum pricing [7], with a centralized mediating entity (CME) acting as a spectrum broker/negotiator to facilitate spectrum sharing among WSPs [4], [6] and/or WSPs and SUs [5].

The cooperation among WSPs from the spectrum sharing perspective is a requirement in many models that are based on short-term spectrum leasing [4], [5], [8]. Some models [4], [9], [10] use the CME to obtain spectrum on short- or longterm leases from the coordinated access band (CAB), where the $\mathrm{CAB}$ is a collection set of the various unutilized parts of the licensed spectrum band (e.g., public safety band and others). Another model [6], based on the cognitive spectrum channel for transmission of spectrum mechanism, has also received significant attention; however, this model is based on the CME and, in some cases, may require WSP cooperation. Reference [11] presents a traffic-based analysis for interoperator spectrum sharing in universal mobile telecommunication system networks and also involves a CME-like element in their framework, but it does provide an insight into the complexity and the costs involved in the implementation of such frameworks.

A parallel line of research has been SU charging and WSP spectrum pricing with various models based on biddingauctioning mechanisms and game-theoretic approaches 
developed in recent years [12]. Bidding and auctioning mechanisms using CME have been a common theme in the literature as in [5], [10], and [13]; however, these mechanisms would need a CME and are based on a combination of spectrum acquisition and SU access provisioning, thus increasing complexity. Reference [7] provides a good overview of the various game-theoretic approaches that can be applied to SU pricing considering the WSP market; however, in contrast with the proposed framework, most of the market structures presented in this work involve inter-WSP cooperation and the use of the CME-based mechanisms.

Although the aforementioned models based acquiring additional spectrum on a short- or long-term basis can help increase the capacity for the WSPs, the WSPs may require configuration changes in their core and access networks and additional hardware to support more SUs. Considering the revenue gained from SU access on one hand and the investment, infrastructure costs, and possible legal problems due to WSP cooperation on the other, inter-WSP spectrum sharing may not turn out to be a viable option to the WSPs.

From the network architecture perspective for SU access, we observe the following hierarchy:

- Spectrum acquisition aspect: Models primarily focused on the spectrum acquisition aspect based on license transfers, where inter-WSP spectrum sharing or from a CAB requires the $\mathrm{CME}$ as the essential component;

- Operational aspect: Models dealing with the provisioning and the operational aspect of SU access, considering interference avoidance to PUs.

For a complete DSA architecture, the WSPs would need to implement both aspects, since the spectrum acquisition aspect is focused on the licensing of an additional spectrum, which complements the operational aspect focused on the provisioning of DSA.

It must be noted that the framework proposed in this paper exclusively falls under the operational aspect and, as previously indicated, can be considered as an intermediate step toward the complete DSA architecture. Additionally, we would also like to emphasize that a direct quantitative comparative analysis of our framework with other models in the literature cannot be made due to the substantially different approaches and parameters involved.

\section{BASE STATION-CENTRIC FRAMEWORK AND SECONDARY USER-INITIATED NETWORK SELECTION}

Considering the spatial and temporal traffic fluctuations, this framework is designed to improve the efficiency in utilization of spectrum resources in the temporal domain at a BS. In simple terms, the BS will advertise and sell spectrum resource in the form of a service (i.e., voice or data) for a fixed fee per quota, where quota refers to time duration or volume data depending upon the service type. Since every BS will advertise such services, the SU will need to analyze the SU price and select the appropriate BS in the area for temporary wireless access. Assuming that there could be multiple BSs (with same or different RATs) belonging to the same or different WSPs in the area, we have designed the BS as the entity

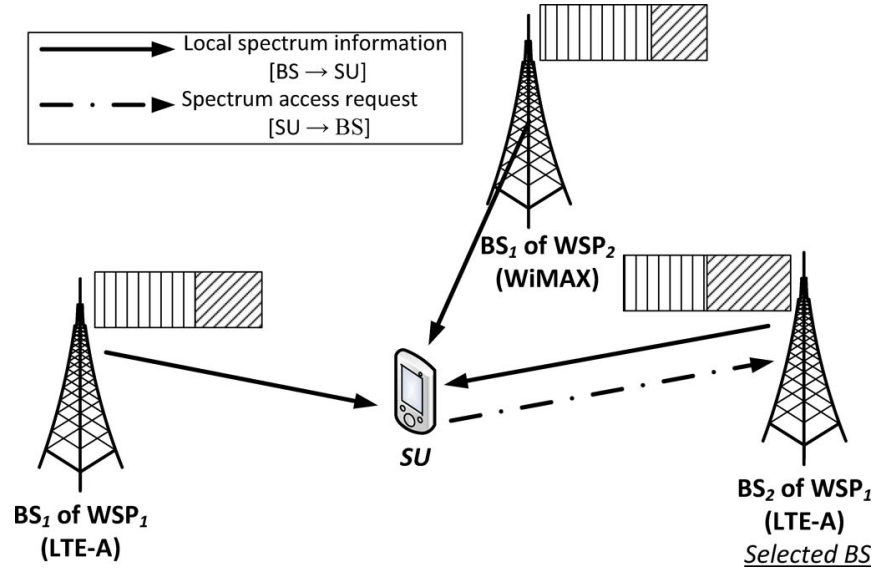

Fig. 2. SU receiving spectrum advertisements from multiple networks (BSs) in the coverage area.

for selection (rather than the WSP), since each BS, depending on the PU demand, would quote a different price for SU access.

\section{A. BS-Centric Framework for SU Access}

Fig. 1 shows a snapshot of the licensed spectrum available at $\mathrm{BS}_{i}$ of $\mathrm{WSP}_{w}$ and its utilization at time instant $t_{x}$. Out of the total spectrum band allotted to $\mathrm{BS}_{i}$, the amount of spectrum currently utilized by the PUs is denoted by $\alpha_{i, w}^{\mathrm{pu}}$, whereas the remaining spectrum that can be potentially made available to $\mathrm{SU}$ is denoted by $\alpha_{i, w}^{\mathrm{su}}=\left(1-\alpha_{i, w}^{\mathrm{pu}}\right)$, where $\alpha_{i, w}^{\mathrm{pu}}, \alpha_{i, w}^{\mathrm{su}} \geq$ 0 . This BS-centric approach is the basis for the proposed framework, which is aimed at enabling the WSPs to generate additional revenue by selling the unutilized spectrum to SUs in real time, if and only such unutilized spectrum is available (i.e., $\alpha_{i, w}^{\mathrm{su}}>0$ ) [14].

A typical scenario of an SU requiring temporary wireless access located in the coverage area of three BSs of same or different WSPs is shown in Fig. 2. On receiving the advertisement with spectrum-related information from each BS in the SU range, the SU will need to analyze the data, select the preferred BS, and then negotiate the parameters for SU access. Section VI, which describes the implementation of the proposed framework to LTE architecture, presents the signaling structure for SU-BS communication with the details related to the signaling information and the parameters that would need to be exchanged and analyzed.

In summary, the BS-centric framework is based on the following features:

1) real-time on-demand temporary wireless access for SUs;

2) harmonious coexistence of PUs and SUs on similar power levels at the same BS, where PU service is not affected by SU access;

3) generation of additional revenue for the WSPs per BS from such SU access;

4) flexibility to the SUs to select or handoff to their preferred BS of any WSP for obtaining temporary wireless access;

5) minimal enhancements required for implementation to the current LTE architecture. 
SUs are assumed to be equipped with multiband user terminals that need the ability to support the multiple RATs to be able to potentially receive SU access or handoff to any BS in a nonrestrictive manner. As previously indicated, the SU terminals are not required to possess cognitive capabilities for spectrum sensing and/or interference management.

Although the proposed framework is not designed to distinguish between types of SUs, we classify the SU into two categories for clarity.

- Type-1 SUs: These SUs are unsubscribed SUs not associated with any WSP in the region;

- Type-2 SUs: These SUs are defined as PUs subscribed with a WSP in the region, with intent to gain temporary access as SUs for a particular application class to which they are not subscribed. For example, PUs subscribed to only "voice" service with "WSP A" could gain temporary access for "data" service through "WSP B" or even "WSP A," (whichever WSP provides the service at a cheaper price)

The definition of Type-2 SUs must not be confused with "roaming" service provided by WSPs, since roaming allows subscribers of a WSP to access a foreign WSP in a different region and requires predefined inter-WSP roaming service agreements. Next, we present the SU-initiated BS selection criterion along with a price-based handoff scheme.

\section{B. SU-Terminal-Initiated Network (BS) Selection Criterion}

Based on the local spectrum utilization, the SUs can select the most appropriate BS based on the spectrum information transmitted by BSs in their coverage area, as shown in Fig. 2.

For this BS selection, the SU would consider the following aspects:

- application class/classes required by the SU and price per application class;

- RATs supported on the SU terminal and those offered by the BSs in the area;

- signal strength recorded at the SU terminal.

Note that the BS selection criterion proposed here is intended to be an autonomous process conducted by the SU terminal, based on the application class input by the SU.

This novel dynamic SU pricing model described in Section VI is dynamic and responsive to PU demand; therefore, the spectrum information (price to be charged to SUs for temporary access at that time instant) is a representation of the localized spectrum information at that BS.

Before proceeding to the BS selection criterion, we introduce the following list of notations:

$s_{i, w}^{j} \quad$ Price charged to the $\mathrm{SU}$ by the $\mathrm{BS}_{i}$ of $\mathrm{WSP}_{w}$ for application class $j$.

$\eta_{i, w} \quad$ Average link spectral efficiency observed at the SU terminal, considering adaptive modulation and coding as given in [15].

$A_{i, w}^{o} \quad$ Application classes offered by $\mathrm{BS}_{i}$.

$A_{r} \quad$ Application classes requested by the SU.

$R_{i, w} \quad$ Set of RATs $x$ supported by the $\mathrm{BS}_{i}$ of $\mathrm{WSP}_{w}$.

$\mathcal{R}_{\mathcal{S}} \quad$ Set of RATs $x$ supported by the SU terminal.
$I_{i, w}^{j} \quad$ Binary integer variable for checking the availability of the $j$ th application class requested by the SU with those offered by the $i$ th $\mathrm{BS}$ of $\mathrm{WSP}_{w}$.

$B_{i, w}^{x} \quad$ Binary integer variable for verifying the support for RAT $x$ provided by the $\mathrm{BS}_{i}$ of $\mathrm{WSP}_{w}$ at the $\mathrm{SU}$ terminal.

A positive integer $M$ with a very large value, e.g., $M \geq$ 1000 , is assigned to the $\mathrm{SU}$ price whenever the application class required by the $\mathrm{SU}$ is not offered by $\mathrm{BS}_{i}$ and is given as

$$
s_{i, w}^{j}=M \text { if } j \in A_{r}, \text { but } j \notin A_{r} \bigcap A_{i, w}^{o} .
$$

The SU-terminal-initiated BS selection criterion can thus be given as follows:

$$
(i, w)^{*}=\left\{\begin{aligned}
B_{i, w}^{x} \times\left(\prod_{j \in A_{r}, j=1}^{n} I_{i, w}^{j}\right) & \\
\times \arg \min _{i=1,2, \ldots, b}\left(\sum_{j \in A_{r}, j=1}^{n} s_{i, w}^{j}\right), & \text { if } \exists !(i, w)^{*} \\
B_{i, w}^{x} \times\left(\prod_{j \in A_{r}, j=1}^{n} I_{i, w}^{j}\right) & \\
\times\left(\arg \max _{i \in B_{s l}}\left(\eta_{i}\right)\right), & \text { otherwise }
\end{aligned}\right.
$$

where

$$
\begin{gathered}
I_{i, w}^{j}= \begin{cases}1, & \text { if } j \in A_{r} \bigcap A_{i, w}^{o} \\
0, & \text { otherwise }\end{cases} \\
B_{i, w}^{x}= \begin{cases}1, & \text { if } x \in R_{i, w} \\
0, & \text { otherwise. }\end{cases}
\end{gathered}
$$

The binary integer variable $I_{i, w}^{j}$ ensures the selection of only those networks where all the application classes requested by the SU are offered by the BSs of the WSPs. The other binary integer variable $B_{i, w}^{x}$ in the objective function ensures that the RAT provided by the BS is supported by the SU terminal. The function in (2) aims to minimize the sum price for all the application classes requested by the SU. It must be noted that if multiple BSs quote the same minimum price, the SU terminal will have to select the BS with the best spectral efficiency from among these shortlisted BSs denoted by the set $B_{s l}$.

\section{SU-Terminal-Initiated Pricing-Based Handoff}

In wireless networks, the handoff mechanism is used to transfer an ongoing subscriber session (voice or data) from the originating BS to the target BS without service interruption. This handoff is primarily triggered when the received signal strength for the originating BS goes below a certain threshold value, while the signal strength for the target $\mathrm{BS}$ is above the threshold value.

In the case of unsubscribed SUs, the affiliation to the WSP is temporary and subject to exhaustion of the allocated quota. During this time period, the WSP would need to consider this $\mathrm{SU}$ as a regular subscriber (short-term PU) and conduct handoffs (if necessary) based on signal strength as in current 


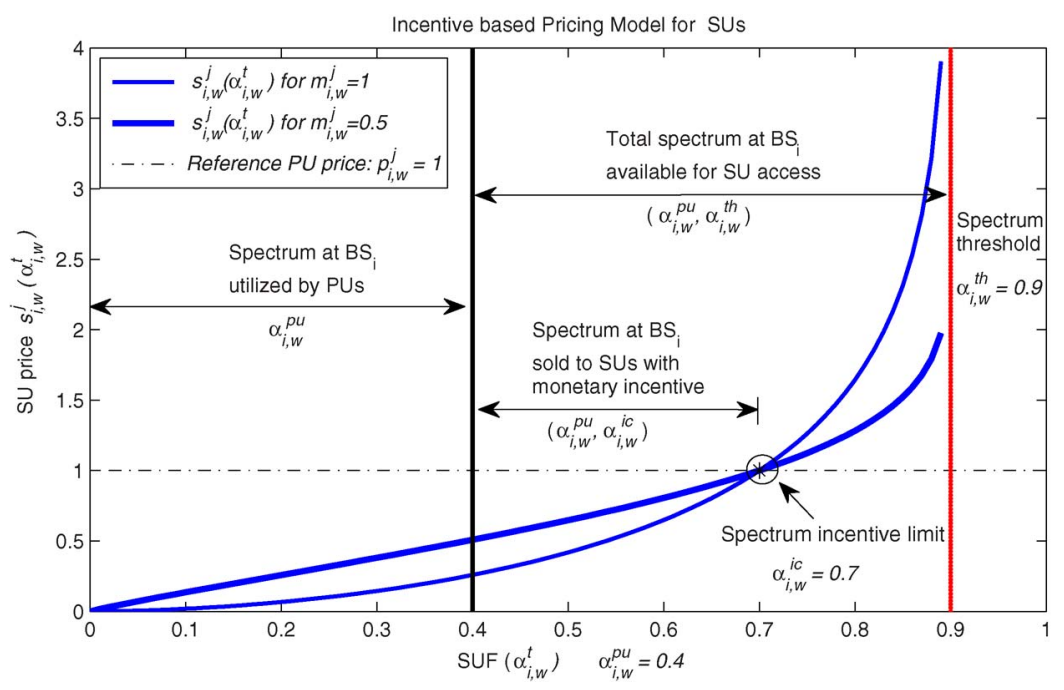

Fig. 3. Dynamic incentive-based SU pricing model. Configuration parameters: $\alpha_{i, w}^{\mathrm{th}}=0.9, \alpha_{i, w}^{\mathrm{ic}}=0.7$.

networks. Finally, at the instant of the SU quota exhaustion, the SU will be disconnected from the WSP. However, the SU may wish to continue to get temporary wireless access from the current WSP or any other WSP in the area. As in the case of the original BS selection, the SU must now check the updated SU price quoted by each BS in the area and trigger a pricebased handoff to the most preferred BS, associated to the same current WSP or a different WSP in the area.

To elaborate the SU-terminal-initiated handoff scheme, we consider a nonreal-time application class "web browsing." Using the criterion for network selection in (2), the SU selects a particular $\mathrm{BS}_{i}$ of a $\mathrm{WSP}_{w}$ to obtain wireless temporary access for a fixed volume data of $B$ bytes of data at time instant $T_{1}$. Assuming that the data transfer of the $B$ bytes requires $T_{2}-T_{1}$ seconds, at time instant $T_{2}$, the temporary service contract for $B$ bytes from $\mathrm{BS}_{i}$ expires, and the new SU price $s_{i, w}^{j}\left(T_{2}\right)$ is calculated considering the new $\mathrm{PU}$ demand at the $\mathrm{BS}_{i}$ of $\mathrm{WSP}_{w}$.

Thus, the SU will have to pay the new SU price $s_{i, w}^{j}\left(T_{2}\right)$ for the next $B$ bytes or could handoff to another BS offering a lower SU price. Once a price-based handoff is triggered using (3), the SU terminal will proceed to scan the F-SUBCH from all the BSs in the area and then, using the network selection criterion in (2), select the most suitable BS.

The information related to $\mathrm{SU}$ prices at time instant $T_{2}$ needs to be provided by the current $\mathrm{BS}_{i}$ of $\mathrm{WSP}_{w}$ well in advance for the SU to trigger a price-based handoff and proceed with network selection, thus continuing the service with minimum interruption. On completion of the handoff, the SU terminal will then be provided temporary access from the newly selected BS. However, improved mechanisms will need to be developed to achieve a seamless price-based handoff, considering the SU authentication procedures.

The price-based handoff will be triggered if the ratio of the difference between the new SU price $s_{i, w}^{j}\left(T_{2}\right)$ and the old (current) SU price $s_{i, w}^{j}\left(T_{1}\right)$ with respect to the old (current) SU price $s_{i, w}^{j}\left(T_{1}\right)$ is greater than the SU-defined cost threshold denoted by $C_{\mathrm{th}}$.
The condition for the price-based handoff can be given as

$$
\frac{s_{i, w}^{j}\left(T_{2}\right)-s_{i, w}^{j}\left(T_{1}\right)}{s_{i, w}^{j}\left(T_{1}\right)} \geq C_{\mathrm{th}}
$$

where $C_{\mathrm{th}}$ is a nonnegative real number. The value of this cost threshold $C_{\text {th }}$ is the only other parameter that needs to be defined by the SUs apart from the application classes required before network selection. The parameter $C_{\text {th }}$ allows for a small amount of hysteresis before the price-based handoff is triggered, thus initiating the network scanning and selection process.

For example, setting $C_{\mathrm{th}}=0.2$ triggers a price-based handoff if there is a $20 \%$ increase in $s_{i, w}^{j}$. However, if $C_{\mathrm{th}}=0$, a price-based handoff will be triggered even if the SU price at time instant $T_{2}$ is slightly greater than the $\mathrm{SU}$ price at time instant $T_{1}$.

The following section will introduce the dynamic incentivebased pricing model for charging SUs, where the dynamic nature is based on the localized spectrum availability at the BS.

\section{Incentive-BAsed PRicing Model FOR CHARging SECONDARY USERS}

This section introduces the incentive-based dynamic pricing model for SUs based on the BS-centric framework described in the previous section. This proposed dynamic SU pricing model has the unique functionality that is aimed at attracting the SUs in periods of low spectrum utilization at the BS by providing them with monetary incentives. The SU pricing model has the inherent property of SU admission control, such that the SUs are gradually discouraged from gaining temporary wireless access owing to the SU price incrementing with every new subsequent $\mathrm{SU}$.

The terminologies for this dynamic SU pricing model shown in Fig. 3 are presented as follows:

- PU utilization $\left(\alpha_{i, w}^{\mathrm{pu}}\right)$ : The PU utilization or PU demand at instant $t_{x}$ at the $\mathrm{BS}_{i}$ of $\mathrm{WSP}_{w}$, where $\alpha_{i, w}^{\mathrm{pu}} \in[0,1]$. 
- SU utilization $\left(\alpha_{i, w}^{\mathrm{su}}\right)$ : The SU utilization or SU demand at instant $t_{x}$ at the $\mathrm{BS}_{i}$ of $\mathrm{WSP}_{w}$, where $\alpha_{i, w}^{\mathrm{su}} \in[0,1]$.

- Spectrum Utilization Factor (SUF) $\left(\alpha_{i, w}^{t}\right)$ : SUF captures the dynamics of the spectrum usage at the $\mathrm{BS}_{i}$ of $\mathrm{WSP}_{w}$ and can be defined as the total spectrum utilization at the $\mathrm{BS}_{i}$ of $\mathrm{WSP}_{j}$, which includes both PUs and SUs being served at time instant $t_{x}$, i.e., $\alpha_{i, w}^{t}=\alpha_{i, w}^{\mathrm{pu}}+\alpha_{i, w}^{\mathrm{su}}$, where $\alpha_{i, w}^{t} \in[0,1]$.

- Reserved spectrum $\left(\alpha_{i, w}^{h}\right)$ : This term represents the spectrum reserved at the $\mathrm{BS}_{i}$ of $\mathrm{WSP}_{w}$ for handoff and/or overload protection, where $\alpha_{i, w}^{h} \in[0,1]$.

- Spectrum utilization threshold $\left(\alpha_{i, w}^{\text {th }}\right)$ : This term represents the total amount of spectrum available for utilization at the $\mathrm{BS}_{i}$ of $\mathrm{WSP}_{w}$ and is based on the reserved spectrum $\left(\alpha_{i, w}^{h}\right)$, denoted by $\alpha_{i, w}^{\mathrm{th}}=1-\alpha_{i, w}^{h}$, where $\alpha_{i, w}^{\mathrm{th}} \in[0,1]$. Thus, the SUs can be provided temporary wireless access if residual spectrum is available at the $\mathrm{BS}_{i}$ of $\mathrm{WSP}_{w}$, i.e., $\alpha_{i, w}^{\mathrm{th}}-\alpha_{i, w}^{\mathrm{pu}}>0$.

- Spectrum incentive limit $\left(\alpha_{i, w}^{\mathrm{ic}}\right)$ : Inherent SU admission control is achieved by penalizing the SUs on the price as the spectrum utilization $\alpha_{i, w}^{t}$ goes beyond a particular limit called the incentive cutoff limit, which the WSPs will set for their respective $\mathrm{BS}_{i}$ of $\mathrm{WSP}_{w}$ and is denoted by $\alpha_{i, w}^{\mathrm{ic}}$, where $\alpha_{i, w}^{\mathrm{ic}} \in[0,1]$. The SUs thus have a monetary incentive $\left(s_{i, w}^{j}<p_{i, w}^{j}\right)$ to obtain wireless access for application class $j$ when $\alpha_{i, w}^{t}<\alpha_{i, w}^{\text {ic }}$, whereas the SUs have a monetary penalty when $\alpha_{i, w}^{t} \geq \alpha_{i, w}^{\text {ic }}$. In the latter case, the SUs need to pay more than the reference PU price $\left(s_{i, w}^{j} \geq p_{i, w}^{j}\right)$ to obtain the same wireless service for application class $j$, thus discouraging the SUs to obtain temporary access.

- Reference PU price $\left(p_{i, w}^{j}\right)$ : This price is the price charged by the WSPs (i.e., $\mathrm{BS}_{i}$ of $\mathrm{WSP}_{w}$ ) for application class $j$ to their subscribers (i.e., PUs). This PU price is the reference price used in the succeeding model to reflect the monetary incentives or penalties charged to the SUs. We have assumed "per minute pricing (in minutes)" for realtime applications and "per fixed data block pricing (unit: MB)" for this reference PU price, and this can be best represented by the "Prepaid/Pay-as-you-go" type services available in current wireless markets.

- SU price $\left(s_{i, w}^{j}\right)$ : This is the price paid by an incoming $\mathrm{SU}$ requesting for wireless access from a particular $\mathrm{BS}_{i}$ of $\mathrm{WSP}_{w}$. Note that although the SU price dynamically changes with respect to spectrum usage variations at $\mathrm{BS}_{i}$, a particular SU will pay a fixed SU price $s_{i, w}^{j}\left(\alpha_{i, w}^{t}\right)$ for temporary access on a per "time duration" or "volume data" basis, whichever applies based on the application class chosen by the SU.

It must be noted that the range of $\mathrm{SU}$ prices will remain constant only for a fixed small time interval $T=T_{f}-T_{s}$ (in minutes), ${ }_{1}^{1}$ where $T_{s}$ indicates the start of the interval and $T_{f}$ the finish. Since the SU price is based on multiple factors (SUF at $\mathrm{BS}_{i}$ of $\mathrm{WSP}_{w}: \alpha_{i, w}^{t} ; \mathrm{BS}_{i}$ configuration parameters: $\alpha_{i, w}^{\mathrm{th}}, \alpha_{i, w}^{\mathrm{ic}}$;

\footnotetext{
${ }^{1}$ Must be configured by the WSP.
}

PU demand: $\alpha_{i, w}^{\mathrm{pu}}$ ), every SU will pay an incremental SU price, but the range of SU prices calculated using (4) will remain frozen for the time interval $T$.

The pricing model that will be presented next also inherently handles SU admission control by dynamically modulating the prices with respect to the fixed reference PU price and based on the PU demand. The pricing for SUs to gain temporary wireless access to application class $j$ during the small time interval $T_{b}-$ $T_{a}$ (in minutes) is given as

$$
\bar{s}_{i, w}^{j}=s_{i, w}^{j}(0)+\left(f_{i, w}^{j}\left(\alpha_{i, w}^{t}\right)\right)^{m_{i, w}^{j}} \times\left(p_{i, w}^{j}-s_{i, w}^{j}(0)\right)
$$

where $s_{i, w}^{j}, s_{i, w}^{j}(0), f_{i, w}^{j}\left(\alpha_{i, w}^{t}\right), m_{i, w}^{j}$, and $p_{i, w}^{j}$ are nonnegative real numbers.

The exponent of $f_{i, w}^{j}\left(\alpha_{i, w}^{t}\right)$ denoted by $m_{i, w}^{j}$ is referred to as the price leveling factor (PLF) and provides additional flexibility to the WSPs to modify their SU pricing curves. The PLF is lower bounded to $m_{i, w}^{j}=0$, since the dynamic SU pricing model becomes static for this case, i.e., $\bar{s}_{i, w}^{j}=p_{i, w}^{j}$. Thus, as $m_{i, w}^{j}$ increases, the range of SU prices $\bar{s}_{i, w}^{j}$ is low (in comparison to the reference PU price) in the interval of SU price incentive $\left(\alpha_{i, w}^{\mathrm{pu}}, \alpha_{i, w}^{\mathrm{ic}}\right)$, whereas $\bar{s}_{i, w}^{j}$ shoots up in the interval of SU price penalty $\left[\alpha_{i, w}^{\mathrm{ic}}, \alpha_{i, w}^{\mathrm{th}}\right)$.

The constant $s_{i, w}^{j}(0)=s_{i, w}^{j}\left(\alpha_{i, w}^{t}=0\right)$ is the initial $S U$ price, i.e., the $\mathrm{SU}$ price at $\mathrm{SUF}=0$, and provides additional flexibility to the WSPs to adjust the dynamic SU price range.

The term $f_{i, w}^{j}\left(\alpha_{i, w}^{t}\right)$ captures the variability of spectrum utilization at the $\mathrm{BS}_{i}$ of $\mathrm{WSP}_{w}$ and can be considered the normalized SU price for application class $j$ with respect to the reference fixed PU price $p_{i, w}^{j}$. This term is based on the log-barrier function [16] and provides inherent SU admission control at $\mathrm{BS}_{i}$ such that

$$
\lim _{\alpha_{i, w}^{t} \rightarrow \alpha_{i, w}^{t h}} f_{i, w}^{j}\left(\alpha_{i, w}^{t}\right)=\infty
$$

thus helping to restrict SU access as the SUF at the BS increases.

The normalized SU price $f_{i, w}^{j}\left(\alpha_{i, w}^{t}\right)$ is defined as follows:

$$
f_{i, w}^{j}\left(\alpha_{i, w}^{t}\right)= \begin{cases}-\ln \left(1-\left(\frac{\alpha_{i, w}^{t}}{\alpha_{i, w}^{\mathrm{th}}}\right)^{n_{i, w}^{j}}\right), & \text { if } 0 \leq \alpha_{i, w}^{t}<\alpha_{i, w}^{\mathrm{th}} \\ \infty, & \text { if } \alpha_{i, w}^{\mathrm{th}} \leq \alpha_{i, w}^{t} \leq 1\end{cases}
$$

where $n_{i, w}^{j}$ is a positive real number representing the exponent in $f_{i, w}^{j}\left(\alpha_{i, w}^{t}\right)$ referred to as the incentive cutoff factor (ICF).

The two parameters $\alpha_{i, w}^{\mathrm{ic}}$ and $\alpha_{i, w}^{\mathrm{th}}$ are the BS configuration parameters and will be set at $\mathrm{BS}_{i}$ by the respective WSP, whereas the $\operatorname{SUF}\left(\alpha_{i, w}^{t}\right)$ is an external parameter and depends on the number of PUs and SUs being served by $\mathrm{BS}_{i}$ of $\mathrm{WSP}_{w}$ at any time instant $t_{x}$. The ICF incorporates all these parameters together into this dynamic pricing model, and its value can be obtained by solving (6) for $\alpha_{i, w}^{t}=\alpha_{i, w}^{\text {ic }}$ and $f_{i, w}^{j}\left(\alpha_{i, w}^{t}\right)=1$.

The functionality of the dynamic SU pricing model can be shown in Fig. 3, where the SU prices are shown to vary with the PU demand and the SUF at $\mathrm{BS}_{i}$ of $\mathrm{WSP}_{w}$ and are based 
on the configuration parameters $\alpha_{i, w}^{\mathrm{th}}=0.9$ and $\alpha_{i, w}^{\mathrm{ic}}=0.7$ and the reference PU price $p_{i, w}^{j}=1$ assuming $s_{i, w}^{j}(0)=0$.

Note that the SU pricing model described here assumes a single BS and a single application class; therefore, considering the geographical area covered by the WSP, the subsequent profits earned from enabling SU access would be manyfold. Next, we extend this dynamic SU pricing model to a multiple WSP environment and present the profitability perspective.

\section{Competitive Secondary User Pricing With Multiple Wireless Service Providers}

Assuming technological feasibility, the successful implementation of any DSA system framework will rely on the potential for profitability to the WSPs in a competitive scenario. This section extends the implementation of the proposed system framework and the SU pricing model to multiple competing WSPs in the SU wireless service market. To develop a competitive SU pricing model with multiple WSPs, a simplified model based on a linear city is considered here. The proposed BScentric framework assumes no cooperation or collusion among the WSPs, as opposed to other models in the literature [7], [12].

Considering two WSPs with different costs and configuration parameters (i.e., $\alpha_{i, w}^{h}, \alpha_{i, w}^{\text {ic }}$ ) implementing a dynamic SU pricing, achieving competitive pricing over a small time interval (e.g., $T=10$ minutes) can become prohibitively complex. This section presents the simplified approach to achieve competitive dynamic SU pricing with two WSPs based on game-theoretic analysis as follows:

- SU service differentiation: The linear city model is used to differentiate SU access based on the wireless channel, i.e., based on the distance of the SU from the BS.

- Equilibrium analysis with static SU pricing: Using this SU service differentiation based on the linear city model, a simple game-theoretic analysis is presented to find the equilibrium SU price based on one fixed SU price. ${ }^{2}$

- Implementation of the dynamic SU pricing model: Finally, the equilibrium SU price is transformed into a dynamic competitive pricing structure using (4).

\section{A. SU Service Differentiation}

Assuming two WSPs competing on price, having identical CAPEX and OPEX costs and providing wireless service for the same application class to PUs, the Nash equilibrium (NE) price per PU can be theoretically shown to be equal to the marginal cost of providing the wireless service, thus providing zero profit for individual WSPs [19]. However, every individual WSP can obtain profits by differentiating their wireless service from other WSPs in the region based on a variety of factors, such as network coverage, brand name, additional subscription features, etc. [17], [19], [20].

Considering the SU market, since the SUs are provided temporary wireless access, the criterion for network (BS) selection can be based on two basic features, namely, the SU price

\footnotetext{
${ }^{2}$ The price charged to the first SU entering the BS, i.e., when the SUF is equal to the PU demand $\alpha_{i, w}^{t}=\alpha_{i, w}^{\mathrm{pu}}$.
}

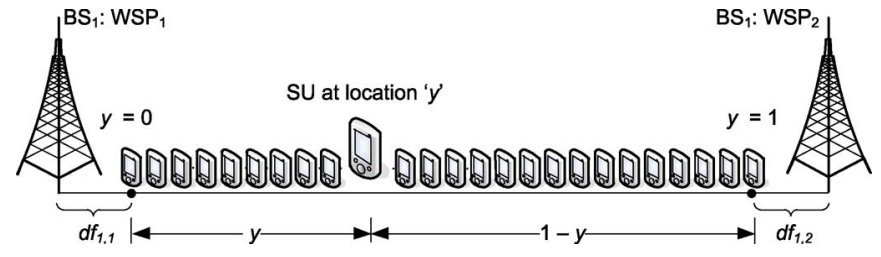

Fig. 4. Linear city model with two WSPs (one BS per WSP).

$s_{i, w}^{j}$ and the average achievable link spectral efficiency $\eta_{i, w}$ estimated based on the signal-to-noise ratio (SNR) observed at the SU terminal, as described in the previous section.

Thus, the differentiation of the SU wireless service based on the location of the SU terminal, which can be modeled in terms of the wireless channel, inherently distinguishes the SU wireless service offered by the BSs of the WSPs in the area.

Next, define the WSP costs and then the linear city model for WSP duopoly with the goal of finding the NE SU price.

1) WSP Costs: The costs borne by $\mathrm{WSP}_{w}$ at a particular $\mathrm{BS}_{i}$ for application class $j$ can be modeled as the sum of the CAPEX denoted by $F_{i, w}^{j}$ and OPEX denoted by $V_{i, w}^{j}\left(\alpha_{i, w}^{t}\right)$. The CAPEX here includes infrastructure and spectrum licensing costs, which are fixed, whereas the OPEX includes operation and maintenance, which are assumed to be variable with respect to spectrum utilization at the BS. The cost to a particular WSP for providing wireless access to SUs through a $\mathrm{BS}_{i}$ of $\mathrm{WSP}_{w}$ for application class $j$ is thus given as

$$
c_{i, w}^{j}=F_{i, w}^{j}+V_{i, w}^{j}\left(\alpha_{i, w}^{t}\right)
$$

where $F_{i, w}^{j}$ and $V_{i, w}^{j}\left(\alpha_{i, w}^{t}\right)$ are positive real numbers. For a fair comparison, the WSP cost is considered per SU and has the same units as the SU price and the PU price. To ensure profitability, the WSP costs $c_{i, w}^{j}$ are upper bounded by the PU price, i.e., $c_{i, w}^{j}\left(\alpha_{i, w}^{t}\right)<p_{i, w}^{j}$.

2) Linear City Model and WSP Duopoly: To differentiate the SU service provided by the two WSPs, a simple scenario with a linear city is considered. A linear city is defined as a city on a line of distance 1 unit, with a single BS per WSP placed on either end at a distance $d f_{i, w}$ outside the city limits, where $d f_{i, w}$ is the exclusion region and depends on antenna configurations at the $\mathrm{BS}_{i}$ of $\mathrm{WSP}_{w}$. The unit distance of the linear city is represented by $y \in[0,1]$ and starts from the exclusion region $d f_{i, w}\left(y=0\right.$ at $\left.d f_{i, w}\right)$ to the cell edge $(y=1)$, as shown in Fig. 4.

To derive the SU demand for the two WSPs, the SUs are assumed to be uniformly distributed along the city, with a single SU at every point $q$ along the line from 0 to 1 . Each of the $q$ SUs is required to select either of the two WSPs for obtaining wireless access for a finite block of $B$ bytes, thus maintaining the demand exactly equal to 1 [19], [20].

To find the NE SU price, we use a fixed SU price $S_{i, w}^{j}$ for every $\mathrm{BS}_{i}$ of $\mathrm{WSP}_{w}$ and fixed identical WSP costs $C^{j}=$ $C_{1,1}^{j}=C_{1,2}^{j}$ for all the SUs in the linear city. This fixed SU price is considered the price for the first $\mathrm{SU}$ entering the BS (i.e., when SUF is equal to PU demand, $\alpha_{i, w}^{t}=\alpha_{i, w}^{\mathrm{pu}}$ ), and SUF is assumed to be in the incentive region, i.e., $S_{i, w}^{j}<p_{i, w}^{j}$ with $\alpha_{i, w}^{t} \in\left(\alpha_{i, w}^{\mathrm{pu}}, \alpha_{i, w}^{\mathrm{ic}}\right)$. From the SU demand standpoint, the BSs 
of both the WSPs are assumed to have sufficient total residual spectrum in $\left(\alpha_{i, w}^{\mathrm{pu}}, \alpha_{i, w}^{\mathrm{ic}}\right)$ to together provide wireless access to all the $q$ SUs in the city.

\section{B. Equilibrium Analysis for Static SU Pricing}

To obtain temporary wireless access from the $\mathrm{BS}_{i}$ of $\mathrm{WSP}_{w}$, the perceived SU price for an SU located at any distance $y$ on the linear city for application class $j$ can be given as

$$
U_{i, w}^{j}(y)=S_{i, w}^{j}+(\zeta \times y)
$$

where $\zeta$ is a constant nonnegative real number representing the "dissatisfaction price" (\$) to an SU, such that the perceived SU price increases as the distance between the $\mathrm{BS}_{i}$ of $\mathrm{WSP}_{w}$ and the SU increases. This dissatisfaction price, which is modeled based on the wireless channel, is described in the next section.

For the SU located at a distance $y$ from the $\mathrm{BS}_{1}$ of $\mathrm{WSP}_{1}$, as shown in Fig. 4, the SU will select $\mathrm{WSP}_{1}$ provided

$$
U_{1,1}^{j}(y)<U_{1,2}^{j}(1-y)
$$

whereas the $\mathrm{SU}$ will select $\mathrm{WSP}_{2}$ if

$$
U_{1,1}^{j}(y)>U_{1,2}^{j}(1-y) .
$$

However, if the two perceived SU prices are found to be equal, i.e., $U_{1,1}^{j}(y)=U_{1,2}^{j}(1-y)$, the selection of the WSPs will be based on a random decision at the SU terminal [18], [19].

It must be noted that the SU prices are assumed to be simultaneously set by each WSP (i.e., neither WSP will have knowledge about the price set by the other WSP), and combined capacity at both the WSPs is able to serve all the $q$ SUs in the linear city. The aforementioned assumptions and the proposed framework ensure that no single WSP can serve all the SUs in the linear city by setting $S_{1,1}^{j}<S_{1,2}^{j}+\zeta$.

Since either WSP cannot gain the entire SU market share by out-pricing the other, the two WSPs will need to set SU prices similar to each other, and the WSPs will need to differentiate the SU service to compete and obtain profits. The following analysis is aimed at finding the NE SU price such that SU prices set by both the WSPs are competitive, with SU service differentiation in guaranteeing individual WSP profits.

Considering an SU located at distance $d$ in the linear city, such that the perceived SU price is equal for either WSP, i.e., $U_{1,1}^{j}(d)=U_{1,2}^{j}(1-d)$, the $\mathrm{SU}$ demand at the $\mathrm{BS}_{1}$ of $\mathrm{WSP}_{1}$ can be given by solving for $d$ as follows:

$$
D_{1,1}^{j}\left(S_{1,1}^{j}, S_{1,2}^{j}\right)=\frac{\zeta-S_{1,1}^{j}+S_{1,2}^{j}}{2 \zeta}
$$

where $D_{1,1}^{j}\left(S_{1,1}^{j}, S_{1,2}^{j}\right)$ is a nonnegative real number and represents the SU demand for the $\mathrm{BS}_{1}$ of $\mathrm{WSP}_{1}$ as a function of the SU prices set by the two WSPs.

The profit for the $\mathrm{BS}_{1}$ of $\mathrm{WSP}_{1}$ from $\mathrm{SU}$ access can thus be given as

$$
\begin{aligned}
\pi_{1,1}^{j}\left(S_{1,1}^{j}, S_{1,2}^{j}\right)=S_{1,1}^{j} \times & \left(D_{1,1}^{j}\left(S_{1,1}^{j}, S_{1,2}^{j}\right)\right) \\
& -C^{j} \times\left(D_{1,1}^{j}\left(S_{1,1}^{j}, S_{1,2}^{j}\right)\right)
\end{aligned}
$$

where $\pi_{1,1}^{j}\left(S_{1,1}^{j}, S_{1,2}^{j}\right)$ is a nonnegative real number and represents the profit to $\mathrm{WSP}_{1}$ from SU access and is a function of the SU prices set by the two WSPs.

With the goal of profit maximization, the best response for $\mathrm{BS}_{1} \mathrm{WSP}_{1}$ to the $\mathrm{SU}$ price set by the $\mathrm{BS}_{1}$ of $\mathrm{WSP}_{2}$ is denoted by $\operatorname{BR}_{1,1}^{j}\left(S_{1,2}^{j}\right)$ and can be given as

$$
\operatorname{BR}_{1,1}^{j}\left(S_{1,2}^{j}\right)=S_{1,1}^{j}=\frac{\partial \pi_{1,1}^{j}}{\partial S_{1,1}^{j}}=\frac{S_{1,2}^{j}+\zeta+C^{j}}{2} .
$$

Assuming the model to be symmetric, a similar set of results can be obtained for the $\mathrm{BS}_{1}$ of $\mathrm{WSP}_{2}$.

Thus, the NE SU price can be expressed as follows:

$$
S^{j *}=C^{j}+\zeta
$$

where $S^{j *}$ is a nonnegative real number representing the NE SU price, assuming the SU prices set by the WSPs to be equal to each other, i.e., $S^{j *}=S_{1,1}^{j *}=S_{1,2}^{j *}$ [19]. It is shown in (14) that the dissatisfaction price " $\zeta$ " (based on the SU location) differentiates the SU wireless service provided by each WSP, thus enhances the profits to both WSPs. This result, therefore, can be easily extended to a multiple WSP environment assuming that the WSP costs and WSP infrastructures are comparable. The aforementioned model and pricing structure can be considered a first step toward the development of more complex analytical models for multiseller pricing, as given in [20].

\section{SU Dissatisfaction Price}

The dissatisfaction price of the SU is defined as a measure of the dissatisfaction of the SU with respect to the signal strength, i.e., SNR, at the SU terminal from the $\mathrm{BS}_{i}$ of $\mathrm{WSP}_{w}$. This parameter is defined as a system parameter and is modeled in terms of the variability of the wireless channel based on the satisfaction level $\mathrm{SL}_{i, w}$ of the SUs in the linear city.

The satisfaction level $\mathrm{SL}_{i, w}(r)$ for the $r$ th $\mathrm{SU}$ in the linear city with respect to the $\mathrm{BS}_{i}$ of $\mathrm{WSP}_{w}$ can be defined as the normalized value of the average achievable link spectral efficiency $\eta_{i, w}(r)$ of the $r$ th SU and is given as

$$
\mathrm{SL}_{i, w}(r)=\frac{\eta_{i, w}(r)}{\max \left(\eta_{i, w}\right)}
$$

where $\mathrm{SL}_{i, w}(r)$ is a nonnegative real number. Assuming shadowing to be averaged out, the maximum value of $\eta_{1,1}$ can be observed at $d f_{1,1}$ (i.e., at $y=0$ nearest to the $\mathrm{BS}_{1}$ of $\mathrm{WSP}_{1}$ ). Thus, the satisfaction level for this $\mathrm{SU}$ (i.e., $\mathrm{SL}_{1,1}$ ) decreases as $y$ increases from 0 to 1 .

The standard deviation of $\mathrm{SL}_{i, w}$ denoted by $\sigma_{i, w}$ can be used to model the variability of the wireless channel considering $q$ SUs uniformly distributed over the linear city and is given as

$$
\sigma_{i, w}=\sqrt{\frac{1}{q-1} \sum_{r=1}^{q}\left(\mathrm{SL}_{i, w}(r)-\overline{\mathrm{SL}}_{i, w}\right)^{2}}
$$

where $\overline{S L}_{i, w}$ denotes the mean satisfaction level for the SUs in the linear city. 
The dissatisfaction price $\zeta$ is based on the wireless channel and is given as

$$
\zeta=K_{1} K_{2} \quad \text { (monetary unit) }
$$

where $K_{1}$ and $K_{2}$ are constants and nonnegative real numbers.

Assuming the monetary price paid by the SU per unit change in $\sigma_{i, w}$ as unity, the two constants $K_{1}$ and $K_{2}$ are defined as follows:

$$
K_{1}=1 \quad \text { (monetary unit) } \quad K_{2}=\left(\frac{\sigma_{1,1}+\sigma_{1,2}}{2}\right) .
$$

Note that the wireless channel, the operating frequency ranges, and the coverage areas for both the WSPs are assumed to be comparable, and hence, this model can be generalized for more than two competing WSPs with comparable parameters. In the generalized case, the value of $K_{2}$ used in calculating $\zeta$ will thus depend on the $\sigma_{i, w}$ 's from all the competing WSPs. Thus, the parameters $K_{1}$ and $K_{2}$ are significant in associating the wireless channel with the pricing structure.

Next, we transform the NE SU price from (14) into a dynamic yet competitive $\mathrm{SU}$ price range.

\section{Implementation of the Dynamic Pricing Model}

The value of the PLF is individually found at the $\mathrm{BS}$ of $\mathrm{WSP}_{i}$ by rearranging (4) and setting $\bar{s}_{i, w}^{j}=S_{i, w}^{j *}$ for $\alpha_{i, w}^{t}=\alpha_{i, w}^{\mathrm{pu}}$ as

$$
\bar{m}_{i, w}^{j}=\frac{\ln \left(\frac{S_{i, w}^{j *}}{p_{i, w}^{j}}\right)}{\ln \left(f_{i, w}^{j}\left(\alpha_{i, w}^{\mathrm{pu}}\right)\right)}
$$

where $S_{i, w}^{j *}$ is the static NE SU price based on (14). As previously described, the PLF maps the NE SU price to the SUF where the first SU enters the system, i.e., $\alpha_{i, w}^{t}=\alpha_{i, w}^{\mathrm{pu}}$.

It must be reiterated that the SU price range and the SU profits for the time interval $T$ further depend on the configuration parameters $\alpha_{i, w}^{\mathrm{ic}}$ and $\alpha_{i, w}^{\mathrm{th}}$ and the average PU demand $\alpha_{i, w}^{\mathrm{pu}}$ before time interval $T$.

Therefore, the aforementioned dynamic SU pricing model not only incorporates the willingness of the SUs to pay, the wireless environment, and the SUF at the BS together in the final price paid by the SUs for temporary wireless access but also guarantees additional SU profits to the WSPs.

\section{E. Simulations and Results}

This section demonstrates the potential for additional profits to the WSPs from SU access based on the proposed framework and the pricing model. It must be noted that the dynamic SU price range and the profits gained are limited to a single BS and for time interval $T$ and, hence, can be further scaled appropriately for annual profit calculations based on the value of $T$ and the total number of BSs deployed by the WSP. Additionally, the results used in the profitability calculations are limited to only those SUs gaining access with a monetary incentive. For simplicity of analysis, SUs paying higher prices than the PU price (monetary penalty region: $\alpha_{i, w}^{t} \in\left(\alpha_{i, w}^{\mathrm{ic}}, \alpha_{i, w}^{\text {th }}\right)$ further boosting additional profits from SUs) have been excluded from these profitability calculations.
TABLE I

WSP PARAMETERS AND RESULTS

\begin{tabular}{|c|c|c|}
\hline \hline Parameter & $\mathbf{W S P}_{1}$ & $\mathbf{W S P}_{2}$ \\
\hline PU utilization $\alpha_{i, w}^{p u}$ & 0.32 & 0.325 \\
\hline Spectrum threshold $\alpha_{i, w}^{t h}$ & 0.9 & 0.85 \\
\hline Incentive cutoff limit $\alpha_{i, w}^{i c}$ & 0.7 & 0.75 \\
\hline Incentive cutoff factor $n_{i, w}$ & 1.83 & 3.67 \\
\hline Results & $\mathbf{W S P}_{1}$ & $\mathbf{W S P}_{2}$ \\
\hline Number of SUs served with monetary incentive & 15 & 18 \\
\hline CP with $\zeta=0.24(\$)$ & 4.5 & 4.9 \\
\hline \hline Competitiveness in CP for $s_{i, w}^{\prime}$ w.r.t. $s_{i, w}$ & 128.99 & 205.98 \\
\hline \hline Comp & $\psi_{s_{1,1}^{\prime}, s_{1,2}^{\prime}: 0.0165}$ & $\psi_{s_{1,1}, s_{1,2}}: 1.6711$ \\
\hline \hline
\end{tabular}

1) Simulation Parameters: The results presented in the following section are based on an urban macrocell scenario [21] with a single cell and no intercell/intracell interference. The following parameters were used for the simulation environment [21]. BS-cell edge distance $=250 \mathrm{~m}$; exclusion distance $d f_{i, w}=35 \mathrm{~m} ; \mathrm{BS}$ total transmit power $=$ $46 \mathrm{dBm} ; \mathrm{BS}$ transmitter antenna gain $=14 \mathrm{~dB}$; SU terminal receiver antenna gain $=0 \mathrm{~dB}$; noise figure $=5 \mathrm{~dB}$; noise power density at $\mathrm{SU}$ terminal $=-174 \mathrm{dBm} / \mathrm{Hz}$ and target $\mathrm{BER}=10^{-6}$. To average over the shadowing experienced by the SUs in the linear city, the spectral efficiencies were calculated from $1000 \mathrm{SU}$ drops and approximated with a third-degree polynomial using least squares curve fitting.

The simulation scenario considers two WSPs with relatively similar average PU demands prior to time interval $T$ (i.e., $\alpha_{1,1}^{\mathrm{pu}} \approx \alpha_{1,2}^{\mathrm{pu}}$ ) with relatively similar yet asymmetrical WSP costs (i.e., $c_{1,1}^{j} \neq c_{1,2}^{j}$ ). The detailed simulation scenario parameters and results for both the WSPs are given in Table I. In addition, to simulate the variability of resource allocation at the BS based on the wireless channel, the SUs are randomly allocated a block of 400,500 , or $600 \mathrm{kHz}$, assuming the total bandwidth available at each BS to be $20 \mathrm{MHz}$.

2) Analysis: Fig. 5 shows SU price competition among two WSPs with different configuration parameters, where the WSP costs are asymmetric, whereas the unutilized spectrum available for SU access is approximately equal, as given in Table I. The significant improvement in profits from SU access after competitive pricing is clearly evident in Fig. 5, where the SU price ranges before and after the application of competitive pricing are displayed. The key observation in Fig. 5 is that the SU price range curves for both WSPs after competitive pricing are highly correlated in spite of the different configuration parameters set by each of the WSPs. Assuming a total of $l$ SUs being allowed access to the $\mathrm{BS}_{i}$ of $\mathrm{WSP}_{w}$ for application class $j$ in the incentive region, the cumulative profit (CP) for the $\mathrm{BS}_{i}$ of $\mathrm{WSP}_{w}$ is a sequence of partial profits denoted by $\mathrm{Pr}_{i, w}^{j}=s_{i, w}^{j}-c_{i, w}^{j}$ and can be given by the following sequence: $\left\{\operatorname{Pr}_{i, w}^{j}(1), \operatorname{Pr}_{i, w}^{j}(1)+\right.$ $\operatorname{Pr}_{i, w}^{j}(2), \operatorname{Pr}_{i, w}^{j}(1)+\operatorname{Pr}_{i, w}^{j}(2)+\operatorname{Pr}_{i, w}^{j}(3), \ldots, \operatorname{Pr}_{i, w}^{j}(1)+$ $\left.\operatorname{Pr}_{i, w}^{j}(2)+\operatorname{Pr}_{i, w}^{j}(3)+\cdots+\operatorname{Pr}_{i, w}^{j}(l)\right\}$. The CPs for both the WSPs before and after competitive pricing are shown in Fig. 6.

To quantify the price competition among the two WSPs, the competitiveness metric $\psi_{s_{1,1}, s_{1,2}}$, which is defined as the 


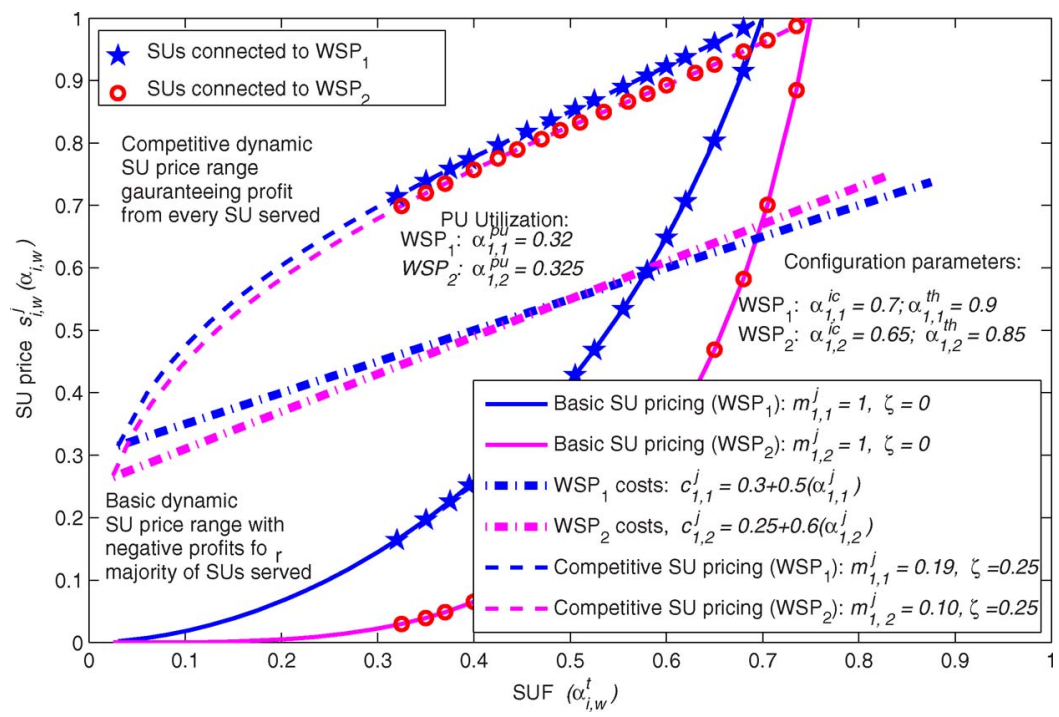

Fig. 5. Competitive SU pricing at the BS between two WSPs.

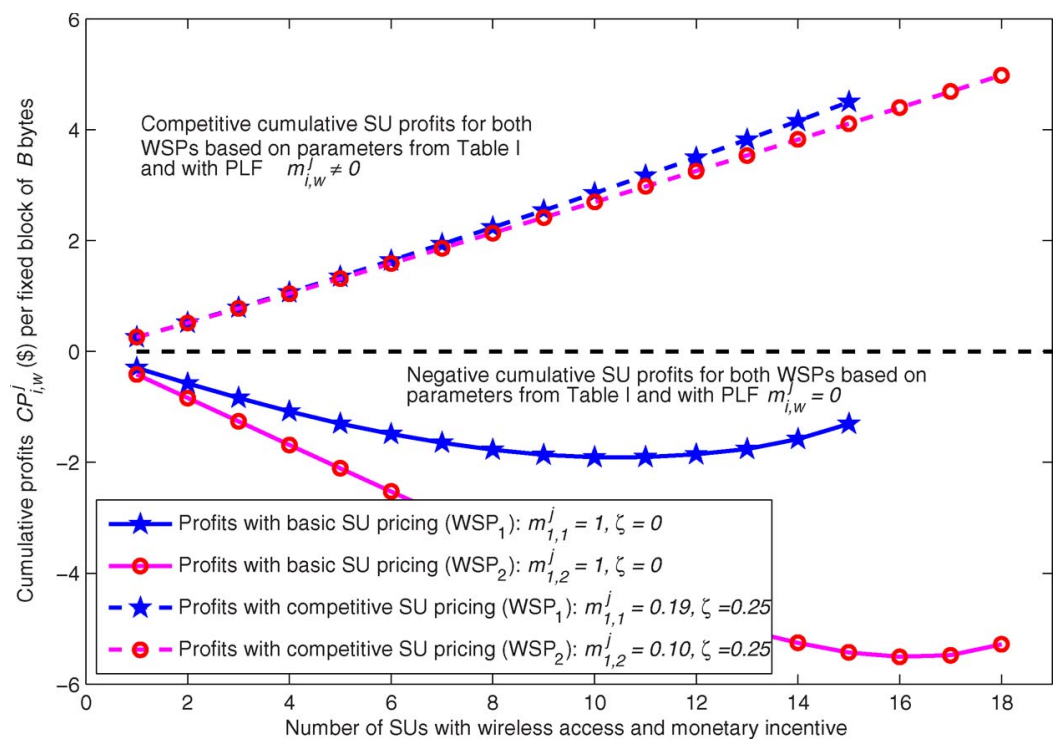

Fig. 6. Competitive profits (CPs) from SU access for the interval $T$.

variance of the difference between the CPs of the competing WSPs, is given as

$$
\psi_{s_{1,1}, s_{1,2}}=\operatorname{VAR}(\Lambda)
$$

where $\Lambda=\left|\hat{s}_{1,1}-\hat{s}_{1,2}\right|$, and VAR denotes the variance.

$\hat{s}_{1,1}=\left\{s_{1,1}(1), s_{1,1}(2), \ldots, s_{1,1}\left(L_{1,1}\right)\right\}$ and $\hat{s}_{1,2}=\left\{s_{1,2}(1)\right.$, $\left.s_{1,2}(2), \ldots, s_{1,2}\left(L_{1,2}\right)\right\}$ give the vector of the original (noncompetitive) $\mathrm{SU}$ prices set by the $\mathrm{BS}_{1}$ of $\mathrm{WSP}_{1}$ and the $\mathrm{BS}_{1}$ of $\mathrm{WSP}_{2}$, respectively, whereas the vector of the new (competitive) SU prices is given by $\hat{s}_{i, w}^{\prime}$. Positive integers $L_{1,1}$ and $L_{1,2}$ represent the number of SUs served by the $\mathrm{BS}_{1}$ of $\mathrm{WSP}_{1}$ and the $\mathrm{BS}_{1}$ of $\mathrm{WSP}_{2}$, respectively. Since either $L_{1,1} \geq L_{1,2}$ or $L_{1,1} \leq L_{1,2}$ such that $q=L_{1,1}+L_{1,2}$, we concatenate either $\hat{s}_{1,1}$ or $\hat{s}_{1,2}$ such that $L_{1,1}=L_{1,2}$ before using them in (20).

The CPs achieved by both the WSPs before and after competitive pricing are plotted in Fig. 6. Similar to the SU price curves in Fig. 5, the CP curves after competitive pricing are highly correlated in comparison to CPs before competition.
This observation can be verified by the competitiveness metric result given in Table I, where the competitiveness metric after competitive pricing denoted by $\psi_{s_{1,1}^{\prime}, s_{1,2}^{\prime}}$ is lower by two orders of magnitude (indicating highly competitive $\mathrm{CPs}$ ), in comparison to the competitiveness metric before competitive pricing denoted by $\psi_{s_{1,1}, s_{1,2}}$. Therefore, we can observe that the lower the value of the competitiveness metric " $\psi$," the higher the competition among the WSPs on prices.

With the dynamic pricing structure for the proposed framework defined, we now present the application of the proposed framework to 4G LTE architecture.

\section{Vi. Application to 4G Long Term Evolution ARCHITECTURE}

This section provides a basic guideline for the implementation of the proposed distributed framework for SU access to the 4G LTE infrastructure, which has been already deployed or is planned for deployment by a majority of the WSPs 


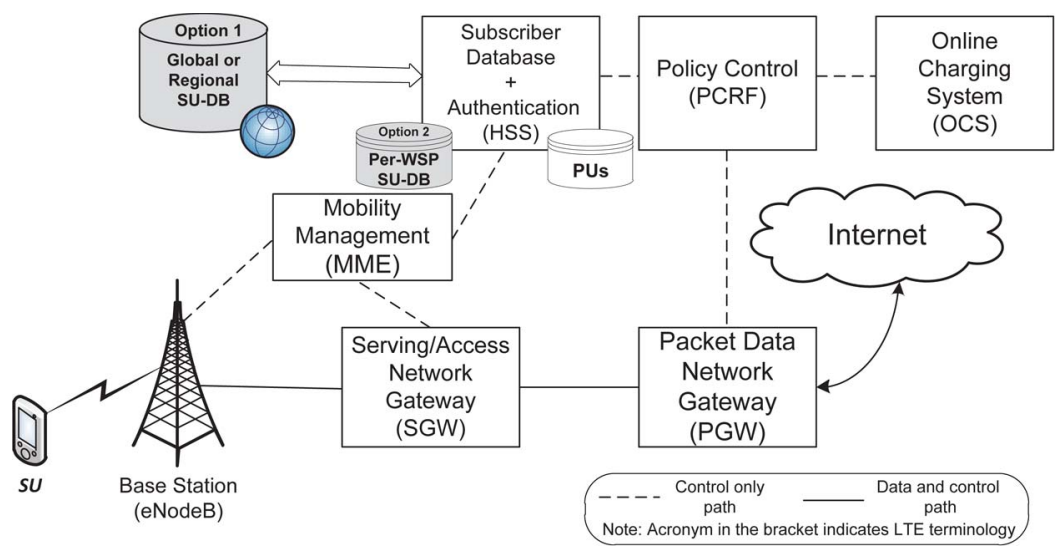

Fig. 7. Infrastructure-based enhancements for enabling SU access (gray boxes) in the 4G LTE architecture.

worldwide. This section will cover the following three aspects: 1) a brief description of the current $4 \mathrm{G}$ LTE architecture, 2) enhancements for enabling $\mathrm{SU}$ access based on the proposed framework, and 3) the SU-BS signaling structure. Fig. 7 shows the basic framework of the 4G LTE architecture, with the proposed enhancements for enabling $\mathrm{SU}$ access depicted in shaded gray.

\section{A. LTE Architectural Components}

The 4G LTE architecture is based on an all-IP framework with backward compatibility to the existing $2 \mathrm{G} / 3 \mathrm{G}$ architectures. Data from the Internet follow the downlink path through the Packet Data Network Gateway (PGW), Serving Gateway (SGW) to the eNodeB ${ }^{3}$ and then to the user equipment (UE). ${ }^{4}$ Here, the PGW provisions the UE IP address, provides a gateway to the Internet, and also manages non-3GPP network handoffs, whereas the SGW manages all the UE data and 3GPPbased network handoffs [22].

The eNodeB is primarily responsible for providing radio resources to the UE based on radio resource management algorithms defined by the WSP. The eNodeB interfaces with the SGW for data plane operations and interfaces with the Mobility Management Entity (MME) for all the control plane activities.

The MME is directly connected to the eNodeB and primarily handles all the control plane functionality in the LTE architecture, which includes UE management, UE location tracking, and handoff. Whenever a UE initiates a service request, the MME communicates with the Home Subscriber Server (HSS) to get subscriber information, associated policies, and authentication details for that UE [22].

Once the HSS authenticates the UE, the PGW acts as the policy enforcer by interfacing with the policy charging and rules function (PCRF), which stores the policies related to application classes provisioned for the UE and the respective data rate limits for that UE. In the LTE architecture UE is the PU. In the proposed model UE can be either PU or SU. The PGW interfaces with the offline charging system for charging regular monthly subscribed PUs and with the online charging

\footnotetext{
${ }^{3}$ Note 1: BSs are referred to as eNodeBs in LTE context.

${ }^{4}$ Note 2: The user handset or subscriber is referred to as UE in LTE context.
}

system (OCS), which is currently useful for charging prepaid PUs based on the duration or volume data [22].

In the following section, we will provide a brief description of the proposed enhancements or modifications to enable temporary SU access in the LTE framework.

\section{B. SU Database and Billing Mechanism}

Since the HSS is responsible for subscriber accounting and authentication, it is essential for an SU requiring temporary wireless access to have their information available at the HSS prior to requesting for $\mathrm{SU}$ access. We therefore propose the addition of a separate database for SUs, i.e., SU database (SUDB), at the HSS.

Since the information at the HSS is unique to every WSP, the SU-DB at the HSS will need to be populated by SU information using either of the two methods as follows:

- Option 1: Global SU-DB: This would be a global SU-DB, where the SUs would need to be registered before they could obtain temporary wireless access. The term global could be relative in meaning here (i.e., regional or nationwide). The HSS will need to have a direct connection to the global SU-DB for acquiring SU information for every new $\mathrm{SU}$ access request.

- Option 2: Local SU-DB: In case the establishment of a global SU-DB is not feasible, WSPs interested in providing temporary SU access need to implement a localized per-WSP database. The SUs will need to register to the localized SU-DB for each WSP separately, prior to requesting SU access from that WSP.

Once the SUs are registered to one of the SU-DBs, they will qualify to obtain SU access to their chosen application class (i.e., voice, data, gaming, etc.) based on the localized spectrum availability (i.e., SUF) at the eNodeBs in the area. As shown in Fig. 7, the HSS is connected to the PCRF, and therefore, the PCRF will have the necessary information to create the required policies for that SU. The PGW, which is connected to the PCRF and the OCS, will then enforce these policies for uplink and downlink data and send the charging information for that SU to the OCS.

Note that although we have presented the incorporation of the proposed framework in the LTE architecture, similar model and 


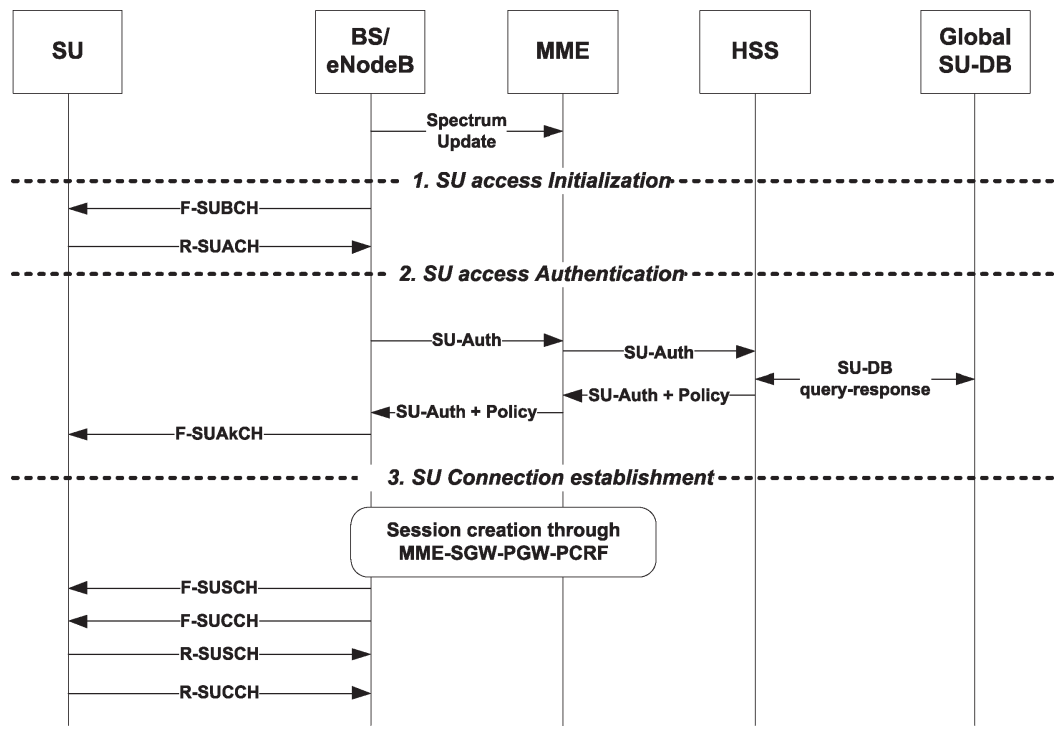

Fig. 8. BS-SU signaling structure for direct temporary SU access.

enhancements could be made for other network architectures (2G, 3G, WiMAX, WiFi, etc.).

\section{BS-SU Signaling Structure}

The previously described architectural components were primarily from the core side of the LTE architecture. Here, we focus on the last mile access side and define the physical channels that would be needed for enabling SU access.

The signaling structure for the SU-eNodeB interaction can be divided into three phases, as shown in Fig. 8, and described as follows.

1) Phase 1-Service Advertisement and BS Selection: In this initialization phase, every eNodeB will need to advertise the following "Spectrum Information Advertisement" message to all the SUs in the area over the Forward-Secondary User Broadcast Channel (F-SUBCH):

- unique identification numbers for eNodeB and their respective WSPs;

- RAT supported by the $\mathrm{BS}_{i}$ of $\mathrm{WSP}_{w}\left(R_{i, w}\right)$;

- application classes offered by the $\mathrm{BS}_{i}$ of $\mathrm{WSP}_{w}\left(A_{i, w}^{o}\right)$;

- price per application class (in monetary units, e.g., \$);

- time interval over which current prices are valid $(T)$;

- frequency of the operation $\left(F_{i, w}^{\mathrm{op}}\right)$;

- frequency for SU response $\left(F_{i, w}^{\mathrm{su}}\right)$.

In addition to the spectrum information advertised by the BS, the received signal strength from each BS is also obtained by the SU terminal. After collection and analysis of the information collected from multiple BSs, the SU will select the most suitable BS based on the BS selection process as described in Section III.

The SU will then send a "Request for Access" message with the following information to the selected BS over the ReverseSecondary User Access Channel (R-SUACH) using the frequency of SU response $\left(F_{i, w}^{\mathrm{su}}\right)$ defined in the advertisement:

- unique SU device identification and SU account details;

- application class requested $\left(A_{r}\right)$;

- RAT supported at the SU terminal $\left(\mathcal{R}_{\mathcal{S}}\right)$.
2) Phase 2-Authentication: As previously indicated, the first step toward gaining temporary wireless access is to register with the SU-DB. The HSS associated with the WSP for the selected $\mathrm{BS}$ will acquire the SU information and conduct the authentication procedure. Based on the result, a positive or negative acknowledgment is then sent to the SUs over the ForwardSecondary User Acknowledgment Channel (F-SUAkCH).

Negative acknowledgment will be sent by the WSP if there is an authentication mismatch, the BS does not have the resources to support the new SU at that instant in time, or the SU does not have enough money (or credit) to proceed with the wireless access.

3) Phase 3-Connection Establishment: Followed by the positive acknowledgment over the F-SUAkCH from the selected BS, the BS will use the Forward-Secondary User Shared Channel (F-SUSCH) for data and Forward-Secondary User Control Channel (F-SUCCH) for control in the downlink, whereas the SUs will use the Reverse-Secondary User Shared Channel (R-SUSCH) for data and ReverseSecondary User Control Channel (R-SUCCH) for control in the uplink.

\section{CONCLUSION}

A techno-economic analysis for $\mathrm{SU}$ access based on the proposed BS-centric distributed framework has been described in this paper. The novel incentive-based dynamic SU pricing model and its extension to a competitive multiple WSP environment present a business case for such SU pricing structures based on the BS-centric distributed framework. From the SUs' perspective, the BS selection criterion and the pricebased handoff scheme provide SUs with the freedom to select or handoff to their preferred BS (i.e., WSP). The strategic model presented in this paper improves the spectrum usage for the WSPs with minimal modifications to the existing infrastructure by providing temporary wireless access to SUs with incentive pricing and it is, therefore, beneficial to both WSPs and SUs. 


\section{REFERENCES}

[1] Rep. Spectrum Efficiency Working Group. Nov. 2002. [Online]. Available: http://www.fcc.gov/sptf/reports.html

[2] Q. Zhao and B. Sadler, "A survey of dynamic spectrum access," IEEE Signal Process. Mag., vol. 24, no. 3, pp. 79-89, May 2007.

[3] J. M. Peha, "Sharing spectrum through spectrum policy reform and cognitive radio," Proc. IEEE, vol. 97, no. 4, pp. 708-719, Apr. 2009.

[4] M. Buddhikot, P. Kolodzy, S. Miller, K. Ryan, and J. Evans, "DIMSUMnet: New directions in wireless networking using coordinated dynamic spectrum," in Proc. IEEE WoWMoM, Jun. 2005, pp. 78-85.

[5] O. Ileri, D. Samardzija, and N. Mandayam, "Demand responsive pricing and competitive spectrum allocation via a spectrum server," in Proc. IEEE DySPAN, Nov. 2005, pp. 194-202.

[6] J. Perez-Romero, O. Salient, R. Agusti, and L. Giupponi, "A novel ondemand cognitive pilot channel enabling dynamic spectrum allocation," in Proc. IEEE DySPAN, Apr. 2007, pp. 46-54.

[7] D. Niyato and E. Hossain, "Market-equilibrium, competitive, and cooperative pricing for spectrum sharing in cognitive radio networks: Analysis and comparison," IEEE Trans. Wireless Commun., vol. 7, no. 11, pp. 4273-4283, Nov. 2008.

[8] S. Shellhammer, "Authorized shared access (ASA)," in Proc. Wireless Innovation Forum: White Space Commun. Summit, Jun. 2011, pp. 1-12.

[9] S. Sengupta and M. Chatterjee, "An economic framework for dynamic spectrum access and service pricing," IEEE/ACM Trans. Netw., vol. 17, no. 4, pp. 1200-1213, Aug. 2009.

[10] J. Jia, Q. Zhang, Q. Zhang, and M. Liu, "Revenue generation for truthful spectrum auction in dynamic spectrum access," in Proc. ACM MobiHoc, May 2009, pp. 3-12.

[11] G. Salami and R. Tafazolli, "Interoperator dynamic spectrum sharing (analysis, costs and implications)," Int. J. Comput. Netw., vol. 2, no. 1, pp. 47-61, Mar. 2010.

[12] C. Gizelis and D. Vergados, "A survey of pricing schemes in wireless networks," IEEE Commun. Surveys Tutorials, vol. 13, no. 1, pp. 126-145, 1st Quart., 2011.

[13] X. Wang, Z. Li, P. Xu, Y. Xu, X. Gao, and H. Chen, "Spectrum sharing in cognitive radio networks-An auction-based approach," IEEE Trans. Syst., Man, Cybern. B, Cybern., vol. 40, no. 3, pp. 587-596, Jun. 2010.

[14] S. Dixit, S. Periyalwar, and H. Yanikomeroglu, "A distributed framework with a novel pricing model for enabling dynamic spectrum access for secondary users," in Proc. IEEE VTC-Fall, Sep. 2009, pp. 1-5.

[15] A. Goldsmith and S.-G. Chua, "Variable-rate variable-power MQAM for fading channels," IEEE Trans. Commun., vol. 45, no. 10, pp. 1218-1230, Oct 1997.

[16] S. Boyd and L. Vandenberghe, Convex Optimization. Cambridge, U.K.: Cambridge Univ. Press, 2004.

[17] R. Barnes, Ecomonic Analysis, An Introduction. London, U.K.: Butterworth, 1971

[18] S. Dixit, S. Periyalwar, and H. Yanikomeroglu, "A competitive and dynamic pricing model for secondary users in infrastructure based networks," in Proc. IEEE VTC-Fall, Sep. 2010, pp. 1-5.

[19] P. K. Dutta, Strategies and Games: Theory and Practice. Cambridge, MA: The MIT Press, 1999.

[20] S. Anderson, A. de Palma, and J.-F. Thisse, Discrete Choice Theory of Product Differentiation. Cambridge, MA: The MIT Press, 1992.

[21] E-UTRA Radio Frequency (RF) system scenarios (Rel. 8), 3GPP TR 36.942 v1.2.0, Jun. 2007.

[22] M. Olsson, S. Sultana, S. Rommer, L. Frid, and C. Mulligan, SAE and the Evolved Packet Core: Driving the Mobile Broadband Revolution. New York: Academic, 2009.

[23] Telecommunication management; Charging management; Charging architecture and principles (Rel. 10), 3GPP TR 32.240 v10.0.0, Dec. 2010.

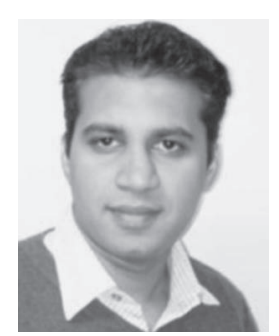

Soumitra Dixit (M'10) received the B.E. degree in electrical and telecommunications engineering from the University of Pune, Pune, India, in 2006 and the M.A.Sc. degree in electrical and computer engineering from Carleton University, Ottawa, ON, Canada, in 2010.

Since 2010, he has been a member of the Technical Expertise Team, Alcatel-Lucent, Ottawa, where he is engaged in internet protocol/multiprotocol label switching (IP/MPLS) routing and the FourthGeneration Long-Term Evolution networks product line. His research interests include wireless communications, IP/MPLS networks, and economic aspects for evolving network architectures.

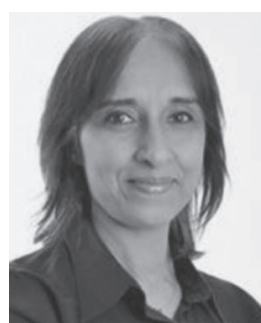

Shalini Periyalwar (M'89-SM'12) received the B.Eng. degree in electrical engineering from Bangalore University, Bangalore, India, in 1981 and the M.A.Sc. and Ph.D. degrees, both in electrical engineering, from Dalhousie University (formerly Technical University of Nova Scotia), Halifax, NS, Canada, in 1987 and 1992, respectively.

During 1991-1994, she held a Nortel-Governmentsponsored Assistant/Research Professor position with the Department of Electrical Engineering, Dalhousie University. During 1994-2006, she held various research positions at Nortel, delivering innovation for supporting business goals. She led teams that contributed radio resource management algorithms to Nortel's North American time-division multiple-access and codedivision multiple-access product evolution. She initiated systems research projects on orthogonal frequency-division multiple access and multihop relays and contributed to the wireless mesh product. Her research contributions have influenced prototype development, enabled standards contributions, and demonstrated innovation to wireless operators. Since 1998, she has been an Adjunct Research Professor with the Department of Systems and Computer Engineering, Carleton University, Ottawa, ON, Canada, where she is cosupervising students on topics addressing next-generation wireless research. During 2006-2010, she was a self-employed Consultant and provided elearning training courses on wireless topics. During 2010-2012, she was a Research Advisor with the Advanced Technology group, Research in Motion, where she continued research on next-generation wireless networks. She is the holder of 40 U.S. patents and has several patents pending. She has written over 30 refereed papers. Her research interests include next-generation wireless solutions including system design, wireless network protocols, and resource management algorithms.

Dr. Periyalwar received the Natural Sciences and Engineering Research Council of Canada Discovery Grant awards from 1992 to 1997 and from 2007 to 2011 .

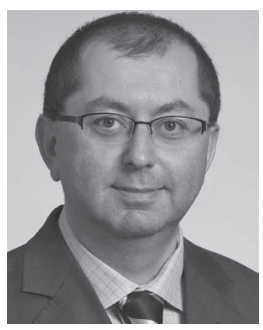

Halim Yanikomeroglu (M'98) was born in Giresun, Turkey, in 1968. He received the B.Sc. degree in electrical and electronics engineering from Middle East Technical University, Ankara, Turkey, in 1990 and the M.A.Sc. degree in electrical engineering and the $\mathrm{Ph} . \mathrm{D}$. degree in electrical and computer engineering from the University of Toronto, ON, Canada, in 1992 and 1998, respectively.

During 1993-1994, he was with the R\&D Group, Marconi Komunikasyon A.S., Ankara. Since 1998, he has been with the Department of Systems and Computer Engineering, Carleton University, Ottawa, ON, where he is currently a Full Professor. He is also an Adjunct Professor with the Advanced Technology Research Institute, King Saud University, Riyadh, Saudi Arabia. During 2011-2012, he was a Visiting Professor with TOBB University of Economics and Technology, Ankara. His research interests include the many aspects of the physical, medium access, and networking layers of wireless communications with a special emphasis on next-generation cellular system. In recent years, his research has been funded by Huawei (Canada and China), Research In Motion (Canada), Samsung (Korea), Communications Research Centre of Canada, Nortel, Natural Sciences and Engineering Research Council of Canada, and Carleton University.

Dr. Yanikomeroglu has been involved in the steering committees and technical program committees of numerous international conferences in communications and has given about 20 tutorials at such conferences. He is a Distinguished Lecturer for the IEEE Vehicular Technology Society. He is a Member of the Steering Committee of the IEEE Wireless Communications and Networking Conference (WCNC), an organization he has been involved with for many years. He served as the Technical Program Cochair of WCNC 2004 and the Technical Program Chair of WCNC 2008 and will serve as the Technical Program Cochair of WCNC 2014, which will be held in Istanbul, Turkey. He was the General Cochair of the IEEE Vehicular Technology Conference Fall 2010 held in Ottawa. He was the Chair of the IEEE Technical Committee on Personal Communications (now called Wireless Communications Technical Committee). He is an Editor for the IEEE TRANSACTIONS ON COMMUNICATIONS. He was an Editor for the IEEE TRANSACTIONS ON WiRELESS COMmunications and the IEEE Communications Surveys and Tutorials. He received the Carleton University Faculty Graduate Mentoring Award in 2010, the Carleton University Graduate Students Association Excellence Award in Graduate Teaching in 2010, and the Carleton University Research Achievement Award in 2009. He is a Registered Professional Engineer in the province of Ontario. 